\title{
Solar cycle and quasi-biennial variations in helioseismic frequencies
}

\author{
Sushanta C. Tripathy ${ }^{1}$, Kiran Jain ${ }^{1}$, Rosaria Simoniello ${ }^{2}$, Frank Hill ${ }^{1}$ \\ and Sylvaine Turck-Chièze ${ }^{2}$ \\ ${ }^{1}$ National Solar Observatory, Tucson, AZ 85719, USA \\ email: stripathy@nso.edu, kjain@nso.edu, fhill@nso.edu \\ ${ }^{2}$ Laboratoire AIM, CEA/DSM-CNRS-I- universite Paris Diderot; CEA, IRFU, SAp, center \\ de Saclay, F-91191, Gif-sur-Yvette, France \\ email: rosaria.simoniello@cea.fr, sylvaine.turck-chieze@cea.fr
}

\begin{abstract}
The prolonged period of solar minimum between cycles 23 and 24 has invoked a great deal of interest to understand the origin of the unusually low solar activity. Even though the origin of solar activity is believed to lie in the shear layer at the base of the convection zone, the analysis of helioseismic data seem to orientate us toward the near surface. In this context, we analyze the signature of the quasi-biennial periodicity seen in the oscillation frequencies which may provide additional constraints on the solar dynamo models.
\end{abstract}

Keywords. Sun: helioseismology - Sun: interior - Sun: oscillations

\section{Introduction}

The investigation of solar oscillation frequencies is providing new insight that may have certain implications for the solar dynamo models. In this context, we examine the variations in intermediate degree oscillation frequencies over a complete solar cycle with emphasis on quasi-biennial periodicity (QBP) which is detected in solar activity indices as well as in oscillation frequencies.

\section{Analysis and Results}

The data analyzed here consist of 171 standard Global Oscillation Network Group (GONG) frequencies computed from 108 day overlapping time series (http://gong.nso. $\mathrm{edu} /$ ) and cover the period 1995 May 7 to 2012 May 24. The mean frequency shifts normalized with mode inertia are determined as the difference between the frequencies of corresponding modes observed on different epochs and the average frequency over the entire period using Eq. (2) of Tripathy et al. (2007). The frequency shifts in the frequency range of $2000 \mu \mathrm{Hz} \leqslant \nu \leqslant 3500 \mu \mathrm{Hz}$ are shown in Fig. 1. It is clear that the short-term variations in the frequency shifts identified as QBP are visible during solar cycle 23 and also during the ascending phase of solar cycle 24 . The dominant 11 year signal modelled in terms of a boxcar filter of width of nearly two years is subtracted from the mean frequency shifts to reveal the residual shifts. The result plotted in top panel of Fig. 1(b) indicates a periodicity of about 2 years as was first noted in low-degree modes by Broomhall et al. (2009) and later extended by Fletcher et al. (2010), Simoniello et al. (2012a) and Jain, Tripathy, \& Hill (2011) for low- and intermediate-degree modes.

In order to quantify the quasi-biennial periodicity in the residual signal, we apply the wavelet analysis developed by Torrence and Compo (1998). The local wavelet power spectrum is shown in bottom panel of Fig. 1(b) where the 2-year QBP can be clearly seen during 1996 through 2007 with a confidence label of $90 \%$ or higher during the 

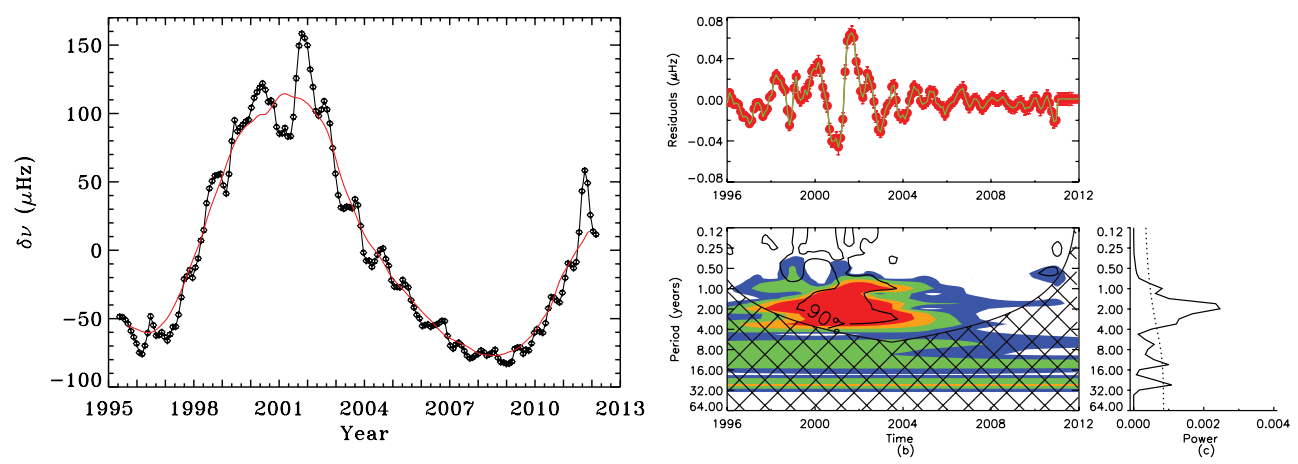

Figure 1. (a) Temporal variation of mean frequency shifts (symbols). The errors in frequency shifts are smaller than the size of the symbols. The solid line represents the dominant 11 year signal of the solar cycle as calculated by applying a boxcar filter of width of roughly two years. (b) The top panel shows the residual shifts after removing the 11 year signal while bottom panel depicts the local wavelet power spectrum, white representing areas of little power, black with the largest power. (c) Global wavelet power spectrum. The dotted line is the $90 \%$ of confidence level.

maximum activity period. A similar result was reported by Fletcher et al. (2010) for low degree modes where the signature of the QBP was also seen during the solar minimum. However we find that the signal is extremely weak during the descending phase of cycle 23. Fig. 1(c) shows the global wavelet power with a peak period of nearly 2 years and its power is higher than $90 \%$ confidence label shown by the dotted line.

Several mechanisms have been proposed to explain the QBP seen in activity indices, such as a second dynamo acting in the sub-surface layers (Benevolenskaja 1998) or instability of magnetic Rossby waves acting in deeper layers (Zaqarashvili 2010). The origin of the acoustic QBP signal is also proposed to be the result of the second dynamo operating at a different depth than the 11 year signal (Fletcher et al. 2010). However, recent analysis using intermediate degree modes argues that the QBP could result from the beating between a dipole and quadrupole magnetic configuration of the dynamo (Simoniello et al. $2012 b$ ). The debate is still open and solar cycle 24 may help to settle some of these issues.

\section{Acknowledgements}

This work utilizes GONG data obtained by the NSO Integrated Synoptic Program (NISP), managed by the National Solar Observatory, which is operated by AURA, Inc. under a cooperative agreement with the National Science Foundation.

\section{References}

Benevolenskaja, E. 1998, ApJ, 509, L49

Broomhall, A.-M., Chaplin, W. J., Elsworth, Y., Fletcher, S., \& Roger, N. 2009, ApJ, 700, L162 Fletcher, S., Broomhall, A. M., Salabert, D., Basu, S., Chaplin, W. J., Elsworth, Y., Garcia, R. A., \& Roger, N. 2010, ApJ, 718, L19

Jain, K., Tripathy, S. C., \& Hill, F. 2011, ApJ, 739:6

Simoniello, R., Finsterle, W., Salabert, D., García, R. A., Turck-Chièze, S., Jiménez, A., \& Roth, M. 2012a, A\&BA, 539, A135

Simoniello, R., Jain, K., Tripathy, S. C., Turck-Chièze, S., Baldner, C. S., Finsterle, W., \& Hill, F, Roth, M. 2012b, ApJ, submitted

Torrence, C. \& Compo, G. 1998, Bull. Am. Met. Soc., 79, 61

Tripathy, S. C., Hill, F., Jain, K., \& Leibacher, J. W. 2007, Solar Phys., 243, 105

Zaqarashvili, T., Carbonell, M., Oliver, R., \& Ballester, J. L. 2010, ApJ, 724, 95 